\title{
INTEGRATION OF $E$-FUNCTIONS WITH RESPECT TO THEIR PARAMETERS
}

\author{
by T. M. MACROBERT
}

(Received 15th January, 1959)

1. Introductory. A number of integrals of $E$-functions with respect to their parameters have been given by Ragab $[1,5]$. Two further integrals of this type are given in $\S 2$ and $\S 3$. In $\S 4$ it is shown that these can be employed to sum series of products of $E$-functions.

The four following formulae will be made use of in the proofs [2].

$$
\begin{aligned}
& \text { If } R\left(\rho_{a+1}\right)>R\left(\alpha_{p+1}\right)>0, \\
& \qquad \begin{array}{l}
\int_{0}^{1} \lambda^{\alpha p+1-1}(1-\lambda)^{\rho_{a+1}-\alpha_{p+1}-1} E\left(p ; \alpha_{r}: q ; \rho_{s}: z / \lambda\right) d \lambda \\
\quad=\Gamma\left(\rho_{a+1}-\alpha_{p+1}\right) E\left(p+1 ; \alpha_{r}: q+1 ; \rho_{s}: z\right) .
\end{array}
\end{aligned}
$$

If $R\left(\alpha_{p+1}\right)>0$,

$$
\int_{0}^{\infty} e^{-\lambda} \lambda^{\alpha p+1-1} E\left(p ; \alpha_{r}: q ; \rho_{s}: z / \lambda\right) d \lambda=E\left(p+1 ; \alpha_{r}: q ; \rho_{s}: z\right) .
$$

If $|\operatorname{amp} z|<\pi$,

$$
E(\alpha:: z)=\Gamma(\alpha)(1+1 / z)^{-\alpha}=z^{\alpha} E(\alpha:: 1 / z) .
$$

If $|\operatorname{amp} z|<\pi$

$$
E\left(p ; \alpha_{r}: q ; \rho_{s}: z\right)=\frac{1}{2 \pi i} \int \frac{\Gamma(\zeta) \Pi \Gamma\left(\alpha_{r}-\zeta\right)}{\Pi \Gamma\left(\rho_{s}-\zeta\right)} z^{\zeta} d \zeta
$$

where the integral is taken up the $\eta$-axis, with loops, if necessary, to ensure that the pole at the origin lies to the left and the poles at $\alpha_{1}, \alpha_{2}, \ldots, \alpha_{p}$ to the right of the contour. Zero and negative integral values of the parameters are excluded and the $\alpha$ 's must not differ by integral values. If $p<q+1$, the contour is bent to the left at both ends. If $p>q+1$, the formula is valid for $|\operatorname{amp} z|<\frac{1}{2}(p-q+1) \pi$.

2. The first integral. If $p \geqslant q$,

$$
\begin{gathered}
|\operatorname{amp} z|<\frac{1}{2}(p-q+2) \pi, \quad R(\delta-\alpha-\beta)>0, \quad R\left(\rho_{n}\right)>R\left(\alpha_{n}\right)>0 \quad(n=1,2, \ldots, q), \\
R\left(\alpha_{n}\right)>0 \quad(n=q+1, \ldots, p), \\
\frac{1}{2 \pi i} \int \frac{\Gamma(\zeta) \Gamma(\alpha-\zeta) \Gamma(\beta-\zeta)}{\Gamma(\delta-\zeta)} E\left(\begin{array}{c}
\delta-\alpha-\beta, \alpha_{1}-\zeta, \ldots, \alpha_{p}-\zeta: z \\
q ; \rho_{s}-\zeta
\end{array}\right) z^{\zeta} d \zeta \\
=\frac{\Gamma(\alpha) \Gamma(\beta) \Gamma(\delta-\alpha-\beta)}{\Gamma(\delta-\alpha) \Gamma(\delta-\beta)} E\left(\begin{array}{c}
\delta-\alpha, \delta-\beta, \alpha_{1}, \ldots, \alpha_{p}: z \\
\delta, \rho_{1}, \ldots, \rho_{q}
\end{array}\right), \ldots \ldots
\end{gathered}
$$

the path of integration being as in (4), with loops, if necessary, to ensure that $\alpha$ and $\beta$ are to the right of the contour.

In proving (5) note that, in virtue of (1) and (2), the integral can be put in the form 


$$
\begin{aligned}
& \frac{1}{2 \pi i} \int \frac{\Gamma(\zeta) \Gamma(\alpha-\zeta) \Gamma(\beta-\zeta)}{\Gamma(\delta-\zeta)} z^{\zeta} d \zeta\left[\prod_{n=1}^{q} \Gamma\left(\rho_{n}-\alpha_{n}\right)\right]^{-1} \prod_{n=1}^{q} \int_{0}^{1} \lambda_{n}^{\alpha_{n}-\zeta-1}\left(1-\lambda_{n}\right)^{\rho_{n}-\alpha_{n}-1} d \lambda_{n} \\
& \times \prod_{n=q+1}^{p} \int_{0}^{\infty} e^{-\lambda_{n}} \lambda_{n}^{\alpha_{n}-\zeta-1} d \lambda_{n} E\left(\delta-\alpha-\beta:: z /\left(\lambda_{1} \ldots \lambda_{p}\right)\right) .
\end{aligned}
$$

Here change the order of integration, putting the first integral last, and get

$$
\begin{aligned}
{\left[\prod_{n=1}^{q} \Gamma\left(\rho_{n}-\alpha_{n}\right)\right]^{-1} \prod_{n=1}^{q} \int_{0}^{1} \lambda_{n}^{\alpha_{n}-1}\left(1-\lambda_{n}\right)^{\rho_{n}-\alpha_{n}-1} d \lambda_{n} } \\
\times \prod_{n=q+1}^{p} \int_{0}^{\infty} e^{-\lambda_{n} \lambda_{n}^{\alpha_{n}-1} d \lambda_{n} \Gamma(\delta-\alpha-\beta)\left(1+\frac{\lambda_{1} \ldots \lambda_{p}}{z}\right)^{\alpha+\beta-\delta}} \\
\quad \times \frac{1}{2 \pi i} \int \frac{\Gamma(\zeta) \Gamma(\alpha-\zeta) \Gamma(\beta-\zeta)}{\Gamma(\delta-\zeta)}\left(\frac{z}{\lambda_{1} \ldots \lambda_{p}}\right)^{\zeta} d \zeta .
\end{aligned}
$$

Now, from (4), the last line is equal to

$$
\begin{aligned}
E\left(\begin{array}{c}
\alpha, \beta: z /\left(\lambda_{\mathrm{t}} \ldots \lambda_{p}\right) \\
\delta
\end{array}\right)=\frac{\Gamma(\alpha) \Gamma(\beta)}{\Gamma(\delta)} F\left(\begin{array}{c}
\alpha, \beta ;-\left(\lambda_{1} \ldots \lambda_{p}\right) / z \\
\delta
\end{array}\right) \\
=\left(1+\frac{\lambda_{1} \ldots \lambda_{p}}{z}\right)^{\delta-\alpha-\beta} \frac{\Gamma(\alpha) \Gamma(\beta)}{\Gamma(\delta-\alpha) \Gamma(\delta-\beta)} E\left(\begin{array}{c}
\delta-\alpha, \delta-\beta: z /\left(\lambda_{1} \ldots \lambda_{p}\right) \\
\delta
\end{array}\right) ;
\end{aligned}
$$

and, on applying (1) and (2), the result is obtained.

Note. The restrictions on the $\rho$ 's can be omitted, as the paths of integration from 0 to 1 can be replaced by contours starting from 0 , passing round 1, and returning to 0 .

3. The second integral. If $p \geqslant q$, $|\operatorname{amp} z|<\frac{1}{2}(p-q+2) \pi, R\left(\alpha_{n}\right)>0(n=1,2, \ldots, p)$,

$$
\begin{aligned}
& \frac{1}{2 \pi i} \int \Gamma(\zeta) \Gamma(\beta-\zeta) E\left(\gamma, \alpha_{1}-\zeta, \ldots, \alpha_{p}-\zeta: q ; \rho_{s}-\zeta: z\right) z^{\zeta} d \zeta \\
&=B(\beta, \gamma) E\left(\beta+\gamma, \alpha_{1}, \ldots, \alpha_{p}: q ; \rho_{s}: z\right),
\end{aligned}
$$

where the path of integration is that of (4), with a loop, if necessary, to ensure that $\beta$ lies to the right of the contour.

The integral is equal to

$$
\begin{aligned}
\frac{1}{2 \pi i} \int \Gamma(\zeta) \Gamma(\beta-\zeta) z^{\zeta} d \zeta\left[\prod_{n=1}^{q} \Gamma\left(\rho_{n}-\alpha_{n}\right)\right]^{-1} \prod_{n=1}^{q} \int_{0}^{1} \lambda_{n}^{\alpha_{n}-\zeta-1}\left(1-\lambda_{n}\right)^{\rho_{n}-\alpha_{n}-1} d \lambda_{n} \\
\times \prod_{n=q+1}^{p} \int_{0}^{\infty} e^{-\lambda_{n}} \lambda_{n}^{\alpha_{n}-\zeta-1} d \lambda_{n} E\left(\gamma:: z /\left(\lambda_{1} \ldots \lambda_{p}\right)\right) \\
=\left[\prod_{n=1}^{q} \Gamma\left(\rho_{n}-\alpha_{n}\right)\right]^{-1} \prod_{n=1}^{q} \int_{0}^{1} \lambda_{n}^{\alpha_{n}-1}\left(1-\lambda_{n}\right)^{\rho_{n}-\alpha_{n}-1} d \lambda_{n} \\
\quad \times \prod_{n=q+1}^{p} \int_{0}^{\infty} e^{-\lambda_{n}} \lambda_{n}^{\alpha_{n}-1} d \lambda_{n} E\left(\gamma:: z /\left(\lambda_{1} \ldots \lambda_{p}\right)\right) \frac{1}{2 \pi i} \int \Gamma(\zeta) \Gamma(\beta-\zeta)\left(\frac{z}{\lambda_{1} \ldots \lambda_{p}}\right)^{\zeta} d \zeta .
\end{aligned}
$$

But the last integral is equal to

$$
2 \pi i E\left(\beta:: z /\left(\lambda_{1} \ldots \lambda_{p}\right)\right)
$$

and, from (3), 


$$
E\left(\beta:: z /\left(\lambda_{1} \ldots \lambda_{p}\right)\right) E\left(\gamma:: z /\left(\lambda_{1} \ldots \lambda_{p}\right)\right)=B(\beta, \gamma) E\left(\beta+\gamma:: z /\left(\lambda_{1} \ldots \lambda_{p}\right)\right) .
$$

Hence, on applying (l) and (2), the result is obtained.

4. Summation of series. Special cases of (5) and (6) are

$$
\begin{aligned}
& \frac{1}{2 \pi i} \int \frac{\Gamma(\zeta) \Gamma(\alpha-\zeta) \Gamma(\beta-\zeta)}{\Gamma(\delta-\zeta)} E(\delta-\alpha-\beta, \gamma-\zeta:: z) z^{\zeta} d \zeta \\
&= \frac{\Gamma(\alpha) \Gamma(\beta) \Gamma(\delta-\alpha-\beta)}{\Gamma(\delta-\alpha) \Gamma(\delta-\beta)} E(\delta-\alpha, \delta-\beta, \gamma: \delta: z),
\end{aligned}
$$

where $|\operatorname{amp} z|<\frac{3}{2} \pi, R(\delta-\alpha-\beta)>0, R(\gamma)>0$; and

$$
\frac{1}{2 \pi i} \int \Gamma(\zeta) \Gamma(\beta-\zeta) E(\gamma, \alpha-\zeta:: z) z^{\zeta} d \zeta=B(\beta, \gamma) E(\alpha, \beta+\gamma:: z),
$$

where $|\operatorname{amp} z|<\frac{3}{2} \pi, R(\alpha)>0$.

These may be employed to sum two series given by Ragab.

The first [3] is

$$
\begin{aligned}
\sum_{r=0}^{\infty} \frac{z^{-2 r}}{r ! \Gamma(\gamma+r)} E(\gamma+r, \alpha & +\beta-\delta+r:: z) E(\gamma+r, \delta-\alpha+r, \delta-\beta+r: \delta+r: z) \\
& =\frac{\Gamma(\delta-\alpha) \Gamma(\delta-\beta) \Gamma(\alpha+\beta-\delta)}{\Gamma(\alpha) \Gamma(\beta)} E(\alpha, \beta, \gamma: \delta: z), \ldots \ldots .
\end{aligned}
$$

where $|\operatorname{amp} z|<\frac{3}{2} \pi, R(\alpha+\beta-\delta)>0, R(\gamma)>0$.

To prove this, substitute on the left from (4), so getting

$$
\begin{aligned}
\sum_{r=0}^{\infty} \frac{z^{-2 r}}{r ! \Gamma(\gamma+r)} \frac{1}{2 \pi i} \int & \Gamma(\zeta) \Gamma(\gamma+r-\zeta) \Gamma(\alpha+\beta-\delta+r-\zeta) z^{\zeta} d \zeta \\
& \times \frac{1}{2 \pi i} \int \Gamma(w) \frac{\Gamma(\gamma+r-w) \Gamma(\delta-\alpha+r-w) \Gamma(\delta-\beta+r-w)}{\Gamma(\delta+r-w)} z^{w} d w
\end{aligned}
$$

Here replace $\zeta$ and $w$ by $\zeta+r$ and $w+r$, and change the order of integration and summation. Then the expression becomes

$$
\begin{aligned}
& \frac{1}{\Gamma(\gamma)} \frac{1}{2 \pi i} \int \Gamma(w) \frac{\Gamma(\gamma-w) \Gamma(\delta-\alpha-w) \Gamma(\delta-\beta-w)}{\Gamma(\delta-w)} z^{w} d w \\
& \quad \times \frac{1}{2 \pi i} \int \Gamma(\zeta) \Gamma(\gamma-\zeta) \Gamma(\alpha+\beta-\delta-\zeta) F(w, \zeta ; \gamma ; 1) z^{\zeta} d \zeta .
\end{aligned}
$$

Now apply Gauss's Theorem and get

$$
\frac{1}{2 \pi i} \int \Gamma(w) \frac{\Gamma(\delta-\alpha-w) \Gamma(\delta-\beta-w)}{\Gamma(\delta-w)} E(\alpha+\beta-\delta, \gamma-w:: z) z^{w} d w,
$$

and the result follows from (7).

The second series [4] is 


$$
\sum_{r=0}^{\infty} \frac{z^{-2 r}}{r ! \Gamma(\alpha+r)} E(\alpha+r, \beta+r:: z) E(\alpha+r, \gamma+r:: z)=B(\beta, \gamma) E(\alpha, \beta+\gamma:: z),
$$

where $|\operatorname{amp} z|<\frac{3}{2} \pi, R(\alpha)>0$.

Proceeding as before it is seen that the series is equal to

$$
\begin{aligned}
\frac{1}{\Gamma(\alpha)} \frac{1}{2 \pi i} \int \Gamma(\zeta) \Gamma(\alpha-\zeta) \Gamma(\beta-\zeta) z^{\zeta} d \zeta \frac{1}{2 \pi i} \int \Gamma(w) \Gamma(\alpha-w) \Gamma(\gamma-w) F(\zeta, w ; \alpha ; 1) z^{w} d w \\
=\frac{1}{2 \pi i} \int \Gamma(\zeta) \Gamma(\beta-\zeta) E(\gamma, \alpha-\zeta:: z) z^{\zeta} d \zeta
\end{aligned}
$$

and from (8) the result follows.

\section{REFERENCES}

1. F. M. Ragab, Proc. Glasgonv Math. Assoc., 3 (1956), 94-98.

2. 'T. M. MacRobert, Functions of a complex variable, 4th edition (London, 1954).

3. F. M. Ragab, Proc. Glasgow Math. Assoc., 3 (1958), 194-195.

4. F. M. Ragab, New York University Institute of Mathematical Science, Research Report No. BR-23.

5. F. M. Ragab, Koninkl. Nederl. Akademie van Wetenschappen-Amsterdam, Proc. A. 61 (1958), 335-340.

\section{The Untversity}

GLASGOW 